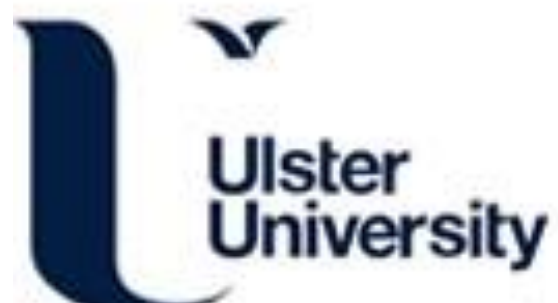

Multi-view fuzzy information fusion in collaborative filtering recommender systems: Application to the urban resilience domain

Palomares, I., Browne, F., \& Davis, P. (2018). Multi-view fuzzy information fusion in collaborative filtering recommender systems: Application to the urban resilience domain. Data and Knowledge Engineering, 113, 6480. https://doi.org/10.1016/j.datak.2017.10.002

Link to publication record in Ulster University Research Portal

\section{Published in:}

Data and Knowledge Engineering

Publication Status:

Published (in print/issue): 31/01/2018

DOI:

10.1016/j.datak.2017.10.002

\section{Document Version}

Author Accepted version

\section{General rights}

Copyright for the publications made accessible via Ulster University's Research Portal is retained by the author(s) and / or other copyright owners and it is a condition of accessing these publications that users recognise and abide by the legal requirements associated with these rights.

\section{Take down policy}

The Research Portal is Ulster University's institutional repository that provides access to Ulster's research outputs. Every effort has been made to ensure that content in the Research Portal does not infringe any person's rights, or applicable UK laws. If you discover content in the Research Portal that you believe breaches copyright or violates any law, please contact pure-support@ulster.ac.uk. 


\title{
Multi-View Fuzzy Information Fusion in Collaborative Filtering Recommender Systems: Application to the Urban Resilience Domain
}

\author{
Iván Palomares ${ }^{\mathrm{a}}$, Fiona Browne, Peadar Davis ${ }^{\mathrm{b}}$ \\ ${ }^{a}$ School of Electronics, Electrical Engineering and Computer Science. Queen's University Belfast. Northern Ireland (United Kingdom). Email: \\ i.palomares@qub.ac.uk \\ ${ }^{b}$ School of Computing and Mathematics. Ulster University. Northern Ireland (United Kingdom). Email: f.browne@ulster.ac.uk
}

\begin{abstract}
Recommender systems play an increasingly important role in on-line web services for the personalization and recommendation of content to individual users. The quantity and quality of user-based information has progressed presenting the opportunity to further tailor recommendations to users based on feature view integration. In this work, we propose a hybrid framework which combines a collaborative filtering recommendation system with fuzzy decision-making approaches (based on the use of aggregation functions) to improve the accuracy of domain-specific recommendations. We extend upon the classical, neighborhood-based collaborative filtering process by conflating preference information with user-profile data in the recommendation process. This is performed using intelligent information fusion techniques whereby Ordered Weighted Averaging (OWA) operators and uninorm aggregation functions are implemented in the fusion of multiple views of pairwise similarity degrees between users. To address the shortcoming of generating sensible recommendations to cold users, we incorporate a novel weighting scheme based on fuzzy set modeling within the uninorm-based aggregation of similarity views. We finally outline the application of the proposed approach through an empirical study based in the Urban Resilience domain, along with an example to movie recommendation.
\end{abstract}

Keywords:

Collaborative filtering recommender systems, multi-view similarity information fusion, fuzzy aggregation, ordered weighted averaging, uninorm, urban resilience

\section{Introduction}

As the availability of digital information, resources and on-line content continues to increase, users have access to a wealth of information. The sheer variety and volume of content available however can make it difficult to find information that most meet their needs. Recommender systems (RS) have gained traction over a number of decades as the go-to approach to address the problem of information overload. These systems aid in information extraction, filtering and predicting relevant information for users [1,2]. One of the most consolidated RS design approaches is that of Collaborative Filtering Recommender Systems (CFRS) which analyse information on users preferences to predict recommendations for them based on their similarity to other users. CFRS are used in various fields, ranging from commerce to financial services, to recommend items of interest to users $[3,4,5]$. Key social media and commercial examples include Twitter, Facebook, Amazon and eBay. Innovative applications of RS techniques include, for instance, social-aware recommendations of conferences [6].

RS solutions have traditionally focused on the preferences of users and the characteristics of items when making their recommendations. This has been driven by the type of data available to RS such as web access logs recording the interaction activity between users and items [7]. As systems have progressed and improved in terms of sophistication, the quantity and quality of feature data available to RS to make recommendations has also improved dramatically $[3,8]$. The integration of multiple relevant features or views is a promising approach to improve the accuracy of domain-specific recommender systems. How to "intelligently" aggregate such domain-specific information from these multiple views is the focus of this paper. To date, there has been limited research focusing on multi-view methods in the context of RS [9, 10]. Initial studies include Oufadia et al. [10], where a recommendation method is presented which combines recommendations generated separately from multiple views, including collaborative, social 
and semantic views. In the study by Guo et al. [9], a multi-view clustering method was developed which integrates two views: preference-based similarity and inter-user trust, prior to generating recommendations. In line with the work by Guo et al., we investigate multi-view methods that flexibly aggregate multiple views of (often disparate) user information before delivering the recommendations. Specifically, we focus on the RS application domains where:

- A collaborative filtering process is undertaken based not only on the preferences/ratings of users, but also on information in their user profile, which contains multiple elements or fields of information of a different nature. Unlike other recommender system paradigms that make use of contextual user information [11], our interest lies in intelligently fusing similarity information pertaining users' profile data, and subsequently aggregating it with preference (rating) similarity information.

- Intelligent information fusion techniques are applied to reflect particular cases when two users have both similar preferences and similar profile, and vice versa [12]. Likewise, the fusion method to combine similarity-based information within the user profile has the flexibility to reflect occurrences of relevant information between two user profiles and take the likelihood of such occurrences into consideration.

- There may exist users with few, or even no ratings yet (cold users) [8, 13], and the multi-view fusion method should deal with these situations accordingly to generate sensible recommendations by striking a balance between the user profile view and the user preference view.

To the best of the authors knowledge, there are no multi-view CFRS methods designed to target these application domains by using flexible and sensitive aggregation functions as of yet. To address this, we present an "intelligent", context-aware multi-view fusion procedure integrated within a CFRS model. The novelty of this work is the proposed multi-view aggregation and fusion approach in collaborative filtering that takes into consideration (1) multiple views of user similarity, (2) the use of Ordered Weighted Averaging (OWA) operators and weighted uninorm aggregation functions to enable "intelligent", attitude-driven fusion of user similarity degrees pertaining multiple views of user data, and (3) a method to alleviate the "new user" cold-start problem in the proposed multi-view similarity fusion framework [13]. The work we present is integrated into a Web platform (detailed in Section 2) for its application in the domain of Urban Resilience [14], however, the approach is generalized and applicable to other problem domains.

This work extends the initial framework presented in [12], by introducing a threefold novel contribution:

1. The definition of an instance of OWA operator that dynamically assigns weights to features of the user profiles to be compared and fuses their similarity information, based on the occurrences found between them and the likelihood of such occurrences. An improved and more flexible method to assign OWA weights is introduced, replacing the highly profile-dependent one in [12] to aggregate the two views of user similarity in our proposed model.

2. The use of uninorm functions, whose mixed behavior and full reinforcement property make them suitable. A weighting scheme based on fuzzy set modeling, is introduced and integrated with the uninorm functions (originally non-weighted in [12]) to appropriately weigh similarity views in the cases when cold users are detected.

3. The approach presented in this study integrates fuzzy set theory [15] to model the concept of cold user and deal with its inherent vagueness and uncertainty to ascertain against well-established users in the system. Fuzzy sets have been recently used in other RS domains to model uncertain information [16], with interesting results $[5,13,17]$. However, to our knowledge these works do not involve the use of fuzzy set theory to specifically cope with the "new user" cold-start problem in a multi-view aggregation-based recommender setting.

The article is presented as follows: a summary of the problem domain Urban Resiliance along with CFRS, OWA and uninorm aggregation functions is presented in Section 2. Section 3 presents the multi-view fusion-based CFRS model. A Use Case based in the Urban Resilience domain, the evaluation of the proposed model in such a domain and its applicability to other domains such as movie recommendation, are presented in Section 4. Finally, we draw conclusions and highlight future work in Section 5. 


\section{Background}

\subsection{Urban Resilience and the HARMONISE Platform}

The term Urban Resilience has stemmed from the expansion of the urban environment (towns and cities) and the need to address uncertainties and challenges such as threats from, for example, climate change and terrorist attacks [18]. A key issue in the Urban Resilience domain is to ensure not only the prevention of an incident but to provide a directed response if one should occur. However, the collective gathering of related urban resources such as material and tools providing information on resilience from design guidance to specifications remains difficult as these resources exist across diverse disciplines. These materials are used by communities from planners and urban designers to construction teams, building security personnel and service managers who are often silo-ed from each other and single community focused. To address this issue, the HARMONISE Web platform was developed to centrally host domain specific content for holistic urban resilience. To provide ease of access to such information, a content management service (CMS) and hosted toolkit with a suite of tools for decision support, understanding and awareness of urban security and resilience, was developed. The HARMONISE Web platform [14, 19] was developed as part of the EU FP7 funded HARMONISE project (http://harmonise.eu/) to provide a holistic approach to urban resilience making large scale built infrastructure secure. A technical overview of the HARMONISE platform can be found in [14]. The platform hosts a suite of tools for decision support, understanding and awareness of urban security and the management of an extensive set of heterogeneous material. These include tools, design guidance and specifications designed to aid built environment professionals. Central to the platform is the developed multiview fusion based CFRS model detailed in this paper. A comprehensive RS is required in this platform to share and highlight diverse urban resilience resources available across multiple user communities. The multi-view fusion based CFRS model combines multiple features including information on user preferences and profile to recommend relevant and new urban resilience tools/resources to the end user.

\subsection{Collaborative Filtering Recommender Systems (CFRS)}

RSs attempt to filter items to users, by predicting a rating value for unseen items by such users then filtering and ranking the "best" unrated items in terms of their prediction value. Examples of existing RS techniques include, but are not limited to:

- Content-based: They recommend items that are similar to those positively rated by the user [20, 21].

- Collaborative filtering-based: They recommend items positively rated by similar users to the target user [22, 23].

- Knowledge-based: They suggest items based on inference on the user needs and preferences [24].

- Demographic: They provide recommendations based on the demographic profile of users [25].

Hybrid approaches and Group Recommender System models, such as collaborative filtering and knowledge-based, or collaborative filtering and demographic, have been subject of extensive research in recent years [7, 24]. A RS typically consists of the following three sources of information [26]:

- A set of items, $X=\left\{x_{1}, \ldots x_{l}\right\}$ (e.g. products, services or other information resources), which may be defined by metadata or other type of information about them.

- A set of users of the system, $U=\left\{u_{1}, \ldots, u_{m}\right\}$ who may provide information about themselves, both explicitly (e.g. age, gender, zip code), and implicitly (i.e. preferences over items).

- A set of users' preferences or ratings over the items, $R \subseteq U \times I \rightarrow D$, expressed as a value in a rating domain $D$, indicating the preference or satisfaction degree of a particular user with a specific item.

CFRS are based on similarity between users $[2,27]$. These methods take the users' preferences over items or rankings as input for predicting (recommending) new items that might potentially be of interest to them, based on items positively rated by similar users or (neighbors). The underlying premise is that those items yet unknown to a 


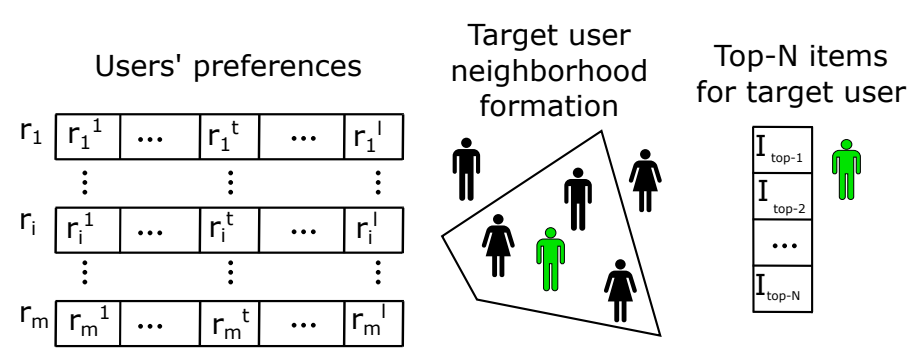

Figure 1: Collaborative Filtering process in RS

target user $u_{i} \in U, i=1, \ldots, m$, and positively rated by similar users, might be foreseen as satisfactory to her/him. There exists an assortment of probabilistic and non-probabilistic approaches for CFRS, such as nearest neighborbased models, dimensionality reduction models, Bayesian models, etc [4]. A common approach in CFRS is the $k$ nearest neighbor $(k N N)$ collaborative filtering, also known as user-user collaborative filtering [28], which determines a neighborhood or subset of similar users to the target user. Central to the neighborhood process in $k N N$-based CFRS, is the use of an adequate similarity measure [22]. The Pearson correlation coefficient, cosine similarity and Spearman rank are well-known examples of similarity measures commonly utilized in related literature. Further detail on similarity measures utilized in our proposal is given in Section 3.1.

Based on the set of neighbor users to $u_{i}$, a prediction function is utilized to predict a rating value for each item not rated yet by $u_{i}$. The most frequently utilized prediction function is a weighted sum function that aggregates neighbor users' ratings over an item by using similarities as weights. As a result, a list of recommendations is delivered by decreasing order of such a prediction value [23]. Figure 1 illustrates the operation scheme of a CFRS. This shows how items are ranked for a target user based on neighborhood formation and user preference similarity.

Users' ratings in CFRSs can take different forms, depending on the system and application domain in which they are implemented [22]. In many domains, numerical ratings such as a 1-5 numerical scale are typically adopted. In the case of the HARMONISE platform, users do not assign ratings to items explicitly, but rather they can optionally mark an item as favorite. This kind of ratings, known as implicit or unary ratings, are common in e-commerce deployments: an item $x_{t} \in X, t=1, \ldots, l$, is either rated by $u_{i}$ (e.g. marked as favorite, purchased in online shops), or not rated (unknown or non-specified preference over $x_{t}$ ). Thus, $u_{i}$ 's preferences are given by the set of items marked as favorite by her/him, $X_{i} \subset I$.

The cold-start problem has been subject of a considerable deal of research within the area of CFRS. This problems arises when the amount of available ratings is relatively small and hence insufficient to effectively apply traditional collaborative filtering techniques [29]. Two main variants of the cold-start problem have been distinguished: the new item cold-start problem, which occurs when a new item has been introduced in the system and not enough users rated it; and the new user cold-start problem, which takes place when a newly registered user has rated a small or null number of items, hence the system is unable to produce meaningful recommendations for her/him [30]. Likewise, different types of methods have been proposed to deal with the issue, such as: making use of additional information sources, improving the user similarity methods, and using hybrid RS methods [13].

\subsection{OWA and Uninorm Aggregation Functions}

The fusion of information is an essential element in intelligent and decision support systems [31]. RS are no exception in applying intelligent aggregation techniques to avail of different sources of information to produce meaningful recommendations [32]. The purpose of aggregation functions is to combine a $n$-tuple of values or elements belonging to a set (e.g. unit interval [33]) into a single representative value.

Definition 1. [33] An aggregation function in the unit interval is a mapping $f:[0,1]^{n} \rightarrow[0,1], n \geq 1$, producing an output value from a set of $n$ input values $A=a_{1}, \ldots, a_{n}$. Every aggregation function in the $[0,1]$ interval satisfies the following three properties:

(i) Identity when Unary: $f(a)=a$.

(ii) Boundary: $f(0, \ldots, 0)=0$ and $f(1, \ldots, 1)=1$. 
(iii) Monotonicity or Non-decreasing: $a_{z} \leq b_{z} \forall z=1, \ldots, n$ implies $f\left(a_{1}, \ldots, a_{n}\right) \leq f\left(b_{1}, \ldots, b_{n}\right)$.

Typically, aggregation in RS has been undertaken to combine similarity and rating information, by applying prototypical functions such as the arithmetic or weighted mean. In some contexts however, it is desirable a function that fulfills additional mathematical properties, for instance:

1. Idempotence: $\mathrm{f}(a, a, \ldots, a)=a$.

2. Compensation: $\min _{z} a_{z} \leq f\left(a_{1}, \ldots, a_{n}\right) \leq \max _{z} a_{z}$.

3. Associativity: $f(a, f(b, c))=f(f(a, b), c)$.

4. Reinforcement: Tendency of multiple high (resp. low) values to reinforce each other, leading to an even higher (resp. lower) result.

For the interested reader, we refer to $[33,34]$ for a comprehensive overview of the main classes of aggregation functions.

The OWA (Ordered Weighted Averaging) operators were introduced by Yager in [35], and they constitute a widely used family of weighted aggregation operators in the literature, particularly in multi-criteria decision support and fuzzy decision making [36]. They are formally defined as follows:

Definition 2. [35] Let $A=\left\{a_{1}, \ldots, a_{n}\right\}\left(a_{z} \in[0,1]\right)$ be a set of $n$ values to aggregate. A OWA operator is a mapping $O W A_{W}:[0,1]^{n} \rightarrow[0,1]$, with an associated weighting vector $W=\left[w_{1} w_{2} \ldots w_{n}\right]^{\top}$, such that $w_{z} \in[0,1], \sum_{z} w_{z}=1$ and,

$$
O W A_{W}\left(a_{1}, \ldots, a_{n}\right)=\sum_{z=1}^{n} w_{z} b_{z}
$$

where $b_{z}$ is the $z$-th largest value in A. OWA operators are characterized by assigning a weight $w_{z}$ to the $z$-th largest element in A, unlike classic weighted average operators, which assign weight $w_{z}$ to the $z$-th element in the input set, $a_{z}$.

The behavior of OWA operators can be flexibly defined and classified based on their weighting vector $W$ (either optimistic, pessimistic or neutral). To determine the attitudinal character of the specific operator utilised, a measure called orness, denoted by orness $(W)$ was also introduced in [35]:

$$
\operatorname{orness}(W)=\frac{1}{n-1} \sum_{z=1}^{n}(n-z) w_{z}
$$

Optimistic (OR-like) OWA operators are those where orness $(W)>0.5$, whereas pessimistic (AND-like) operators have orness $(W)<0.5$ [37]. The higher orness $(W)$, the more importance is assigned to the highest values in $A$, therefore the closer the aggregated result is to $\max \left(a_{1}, \ldots, a_{n}\right)$. Conversely, the lower orness $(W)$, the more importance is given to the highest values in $A$, and the closer the output is to $\min \left(a_{1}, \ldots, a_{n}\right)$.

A central aspect for the definition of an OWA operator consists in the construction of the weighting vector $W$. Different approaches have been proposed in the literature to facilitate their computation, e.g. by using fuzzy linguistic quantifiers or from learning approaches [35, 38]. Some particular cases of OWA operators are [39]:

- The maximum operator, with orness $(W)=1, w_{1}=1$ and $w_{z}=0, z \neq 1$.

- The minimum operator, with orness $(W)=0, w_{n}=1$ and $w_{z}=0, z \neq n$.

- The arithmetic mean, with orness $(W)=0.5$ and $w_{z}=1 / n \forall z$.

Uninorm aggregation operators were introduced by Yager and Rybalov in $[40,41]$ to provide a generalization of the t-norm and the t-conorm operators [33]. Unlike t-norms and t-conorms, whose neutral elements are 1 and 0 respectively, uninorms have a neutral element $g \in[0,1]$ lying anywhere in the unit interval. Whilst OWA operators allowed to define varying attitudes within an averaging behavior, uninorm aggregation operators present a varying behavior (namely conjunctive, disjunctive or averaging), depending on the input values being higher or lower than $g$. They are defined as follows: 
Definition 3. [40] A uninorm is a mapping, $\mathcal{U}:[0,1]^{2} \rightarrow[0,1]$, having the following properties for all $a, b, c, d \in$ $[0,1]$ :

i) Commutativity: $\mathcal{U}(a, b)=\mathcal{U}(b, a)$.

ii) Monotonicity: $\mathcal{U}(a, b) \geq \mathcal{U}(c, d)$ if $a \geq c$ and $b \geq d$.

iii) Associativity: $\mathcal{U}(a, \mathcal{U}(b, c))=\mathcal{U}(\mathcal{U}(a, b), c)$.

iv) Neutral element: $\exists g \in[0,1]: \mathcal{U}(a, g)=a$.

Due to the associativity property, uninorm operators are typically defined for $n=2$, and additional input values can be successively aggregated without affecting the aggregated result. The conjunctive, disjunctive or averaging behavior depends on input values $a, b$ being greater or lower than $g$. This distinctive property is graphically illustrated in Figure 2.

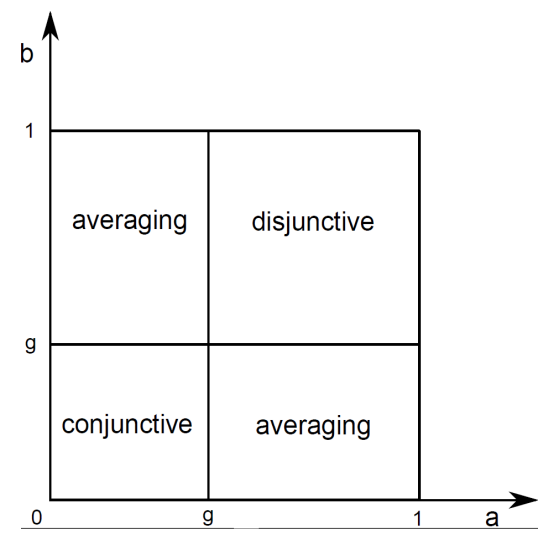

Figure 2: Behavior of uninorm operators for different input values $a, b \in[0,1]$ and a neutral element $g \in[0,1]$

A notable characteristic of uninorm operators is their full reinforcement property: given any $g \in[0,1]$, uninorms show an upward reinforcement when both input values are high (above $g$ ), making the aggregated value even higher (disjunctive behavior). Conversely, they show a downward reinforcement when aggregating low input values (below $g$ ), so that the aggregated value is even lower (conjunctive behavior).

Example 1. The cross-ratio uninorm is a continuous uninorm in $[0,1]^{2} \backslash\{(0,1),(1,0)\}$, with neutral element $g=0.5$ :

$$
\mathcal{U}(a, b)=\left\{\begin{array}{cl}
0 & \text { if }(a, b) \in\{(0,1),(1,0)\}, \\
\frac{a b}{a b+(1-a)(1-b)} & \text { otherwise. }
\end{array}\right.
$$

The concept of weighted uninorm has also been studied in the literature [40, 42]. Generally, the aggregation process followed by a weighted uninorm operator $\mathcal{U}_{W}(a, b)$ consists of two steps: (1) combine each input value $a$ with its weight $w_{a}$ into a single weighted value, $\bar{a}$, and (2) use a uninorm operator to aggregate weighted values, i.e. $\mathcal{U}_{W}(a, b)=\mathcal{U}(\bar{a}, \bar{b})$. Combining inputs with their weights requires a specific type of operator called uninorm weighting operator [42].

Definition 4. Let $\mathcal{U}$ be a uninorm operator with neutral element $g$. A uninorm weighting operator of $\mathcal{U}$ is a mapping $\boldsymbol{w}_{g}:[0,1]^{2} \rightarrow[0,1]$ accomplishing the following properties (with the first argument, a, being the input value, and the second argument, $w$, its associated weight):

(i) If $a \leq b$, then $\boldsymbol{w}_{g}(a, w) \leq \boldsymbol{w}_{g}(b, w), \forall a, b, w \in[0,1]$, i.e. if an input value $a$ is greater or equal than another $b$ and they are both equally important, then its weighted result should not decrease the overall result of aggregation.

(ii) If $w \leq w^{\prime}$, then $\boldsymbol{w}_{g}\left(g^{+}, w\right) \leq \boldsymbol{w}_{g}\left(g^{+}, w^{\prime}\right), \forall w, w^{\prime} \in[0,1], g^{+} \in[g, 1]$, i.e. the higher the weight assigned to an input value higher than $g$, the more (upwards) influence it should play in the overall aggregation.

(iii) If $w \leq w^{\prime}$, then $\boldsymbol{w}_{g}\left(g^{-}, w\right) \geq \boldsymbol{w}_{g}\left(g^{-}, w^{\prime}\right), \forall w, w^{\prime} \in[0,1], g^{-} \in[0, g]$, i.e. the higher the weight assigned to an input value lower than $g$, the more (downwards) influence it should play in the overall aggregation. 


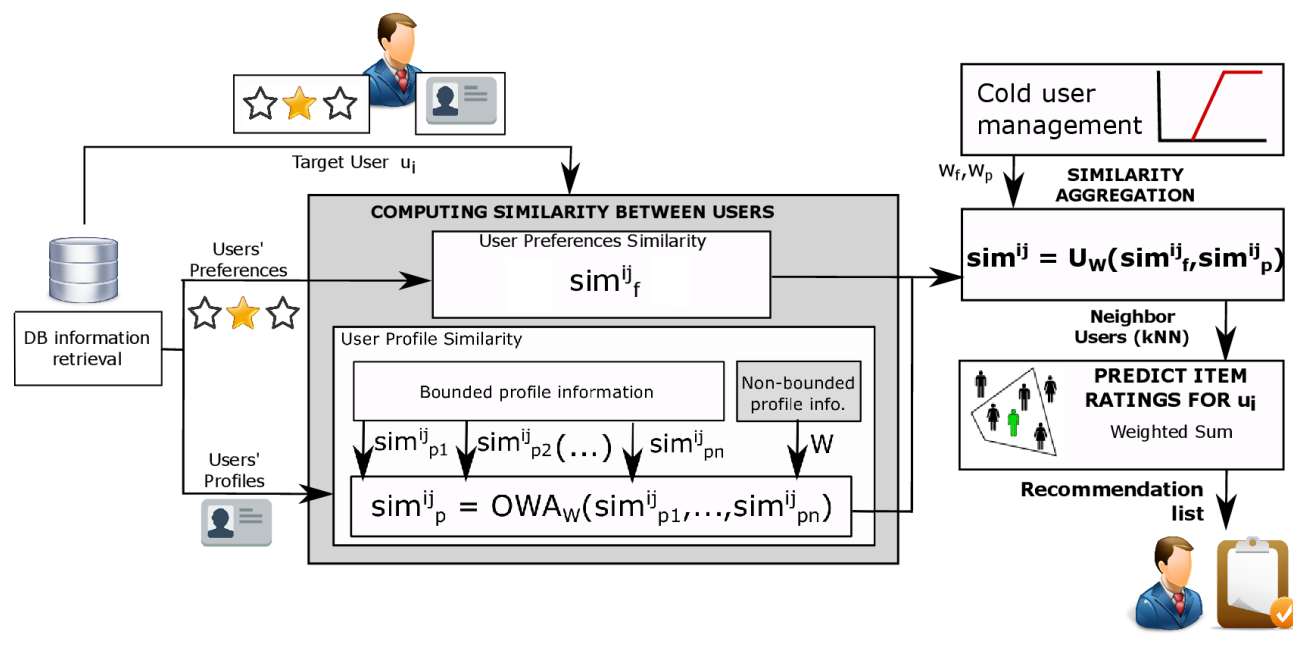

Figure 3: Proposed Multi-View Weighted CFRS model schema

(iv) $\boldsymbol{w}_{g}(a, 1)=a, \forall a \in[0,1]$, implying that when all inputs are equally important and given maximum weight, the uninorm aggregation becomes non-weighted.

(v) $\boldsymbol{w}_{g}(a, 0)=g, \forall a \in[0,1]$, which implies that an input value with null weight will not be taken into account in the overall aggregation.

Example 2. The following is an example of uninorm weighting operator:

$$
\boldsymbol{w}_{g}(a, w)=w(a-g)+g
$$

Following from this overview of aggregation functions we present our proposed methodology of a multi-view fusionbased CFRS model.

\section{Multi-View Fusion-based CFRS Model}

In this section, we present our multi-view CFRS framework that integrates intelligent aggregation functions classically utilized for fuzzy decision-making, to provide improved recommendations to users in diverse domains, such as urban resilience resource sharing across communities. The collaborative filtering model underlying the CFRS incorporates a number of features:

- A multi-view approach to calculate pairwise similarity between users, taking into account both their preferences (given by explicit and/or implicit ratings, depending on the domain tackled) and relevant information featured in their user profile that may indicate potential likeness to other relevant users.

- OWA and uninorm aggregation functions are utilized throughout the recommendation process, for the computation and fusion of (different views of) user similarity degrees. More specifically, a weighting scheme is applied as part of the uninorm aggregation to deal with the "new user" cold-start problem (caused by users with scarce or null preferential information), providing them with sensible recommendations more reliant on user profile information.

- Fuzzy set theory is utilized to model the notion of cold users and classify users as cold, partly cold or wellestablished. This classification will further influence the multi-view fusion and subsequent recommendation processes, as explained in Section 3.4.

The schema depicted in Figure 3 illustrates the methodology of the proposed CFRS model. These phases are summarised in Algorithm 1 and described in detail in the following subsections: calculation of similarity between user preferences (3.1) and profiles (3.2), multi-view similarity aggregation (3.3) and its associated weighting method to manage cold users (3.4), and finally, neighborhood identification and recommendation process (3.5). 


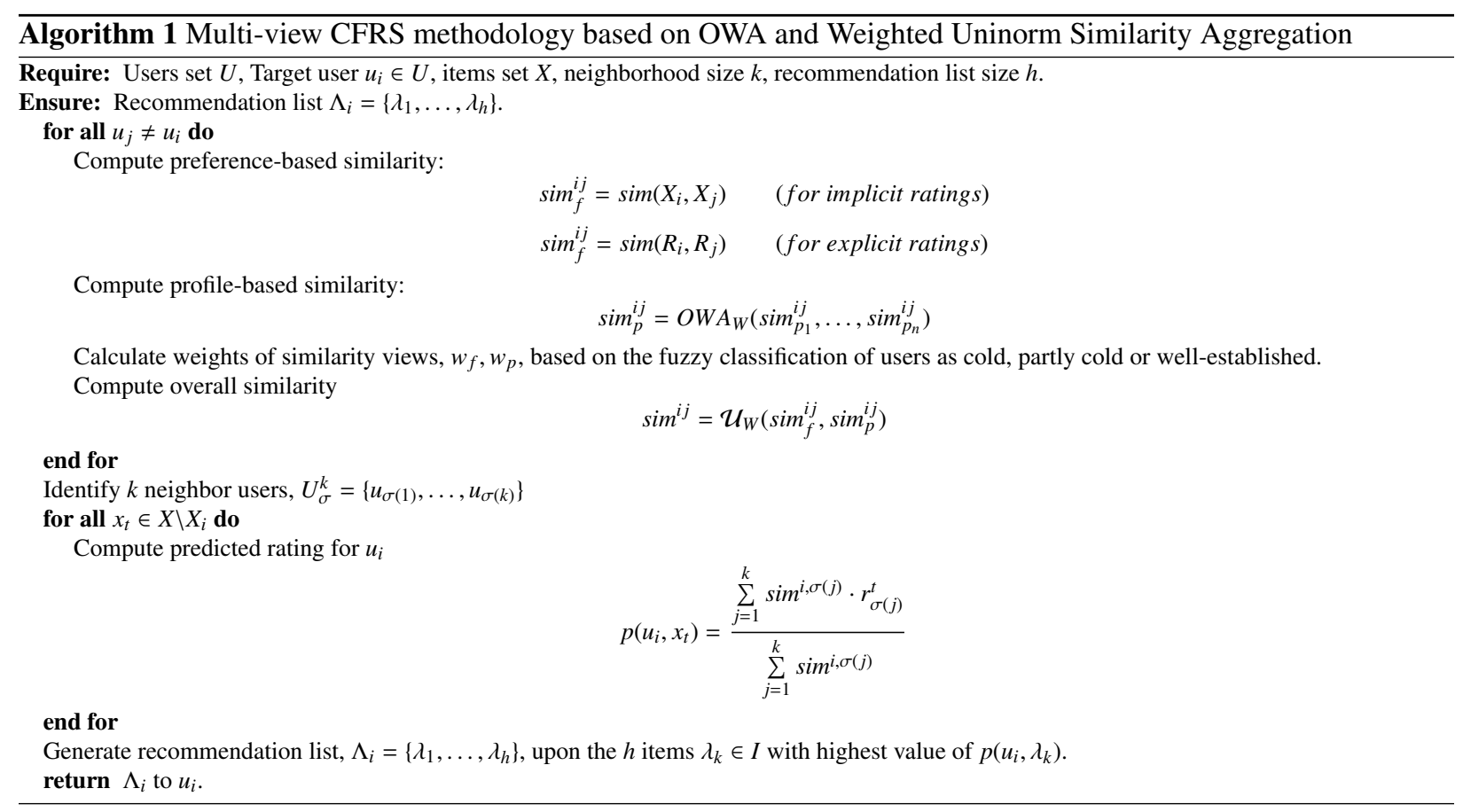

\subsection{Similarity between User Preferences}

The proposed CFRS has been conceived to deal with diverse rating domains, including both explicit (numeri$\mathrm{cal} /$ ordinal) ratings, and implicit (unary) ratings. We now introduce the procedure to calculate the preference-based user similarity for each of these types of rating data.

\subsubsection{Preferences based on Implicit Rating Data}

Implicit rating information, or unary ratings, allow users to specify positive preference for an item via her/his actions (e.g. marking an item as favorite, viewing it repeatedly, etc.), but it cannot reflect degrees of preference or a negative preference [29]. Let $r_{i}^{t}$ denote the rating of a user $u_{i}$ over an item $x_{t}$, such that an item is either rated (marked/classified as favorite by the user). Importantly, in a recommender domain characterized by implicit rating data, an unrated item $x_{t}$ by $u_{i}$ does not necessarily indicate that $u_{i}$ dislikes it, but rather that the user has not explicitly indicated he/she likes it (e.g. because he/she has not viewed it yet). The degree of similarity between preferences of two users $u_{i}$ and $u_{j}$, denoted $\operatorname{sim}_{f}^{i j} \in[0,1]$, can be calculated based on the Jaccard index $\mathcal{J}_{F}^{i j}=\mathcal{J}\left(X_{i}, X_{j}\right)$ between the two sets, $X_{i}, X_{j} \subset I$, of items marked as favorite by $u_{i}$ and $u_{j}$, respectively:

$$
\operatorname{sim}_{f}^{i j}=\left\{\begin{array}{cc}
\mathcal{J}_{F}^{i j}=\frac{\left|X_{i} \cap X_{j}\right|}{\left|X_{i} \cup X_{j}\right|} & \text { if }\left|X_{i}\right|,\left|X_{j}\right|>0, \\
0 & \text { otherwise }
\end{array}\right.
$$

with $\bigcap, \cup$ the set intersection and union operators, respectively, and $|\cdot|$ denoting the cardinality of a set. Intuitively, $r_{i}^{t}=1$ iff $x_{t} \in X_{i}$, and $r_{i}^{t}=0$ otherwise.

Some properties of $\mathcal{J}$ are outlined below:

(i) $\left|X_{i}\right|=\left|X_{j}\right|=0$ in Eq. (5) indicates that both $u_{i}$ and $u_{j}$ are cold users with no registered ratings in the CFRS yet.

(ii) If one of the two user has no ratings, i.e. $\left|X_{i}\right|>0$ and $\left|X_{j}\right|=0$, their intersection is the empty set, hence it also holds $\mathcal{J}_{F}^{i j}=0, \forall\left|X_{i}\right|$.

(iii) If $\left|X_{i}\right|=1$, then $\mathcal{J}_{F}^{i j} \in\left\{0, \frac{1}{\left|X_{j}\right|}\right\}, \forall\left|X_{j}\right|>0$. 
(iv) In general, for any two non-empty sets of favorite items $X_{i}, X_{j}$ such that $\left|X_{i}\right| \leq\left|X_{j}\right|$ and $\left|X_{i j}\right|=\left|X_{i}\right|+\left|X_{j}\right|$, we have:

$$
\mathcal{J}_{F}^{i j} \in\left\{0, \frac{1}{\left|X_{i j}\right|-1}, \frac{2}{\left|X_{i j}\right|-2}, \ldots, \frac{\left|X_{i}\right|}{\left|X_{j}\right|}\right\}
$$

By default, the Jaccard index tends to provide excessively low (hence non-meaningful) values when $X_{i}, X_{j}$ and $X$ are large. This clearly poses an untoward effect in RS domains where a moderate number of coincidences between two users should be viewed as a sign of similarity between them. A possible straightforward solution consists in relaxing the Jaccard index through applying a $\varepsilon$-root function, with $\varepsilon \geq 2$ :

$$
\operatorname{sim}_{f}^{i j}=\sqrt[\varepsilon]{\mathcal{J}\left(X_{i}, X_{j}\right)}
$$

Another option is to utilize the cosine similarity metric, which in the case of unary ratings can be easily expressed in terms of $X_{i}, X_{j}$, as follows:

$$
\operatorname{sim}_{f}^{i j}=\frac{\sum_{t} r_{i}^{t} \cdot r_{j}^{t}}{\left\|\mathbf{r}_{i}\right\|_{2} \cdot\left\|\mathbf{r}_{j}\right\|_{2}}=\frac{\sum_{t} r_{i}^{t} \cdot r_{j}^{t}}{\sqrt{\sum_{t} r_{i}^{t}} \cdot \sqrt{\sum_{t} r_{j}^{t}}}=\frac{\left|X_{i} \cap X_{j}\right|}{\sqrt{\left|X_{i}\right|} \cdot \sqrt{\left|X_{j}\right|}}
$$

where $\left\|\mathbf{r}_{i}\right\|_{2}$ denotes the norm of the vector representation $\mathbf{r}_{i} \in\{0,1\}^{\left|X_{i}\right|}$ of all $u_{i}$ ratings. Without loss of generality, we hereinafter consider $\operatorname{sim}_{f}^{i j}=\sqrt{\mathcal{J}\left(X_{i}, X_{j}\right)}$.

Remark 1. As discussed above, from property (iv) of the Jaccard index-based similarity, if $\left|X_{i}\right| \ll\left|X_{j}\right|$, the resulting similarity degree turns to be low. This occurs even in the "best" scenario when all items in $X_{i}$ are also in $X_{j}$, i.e. $X_{i} \subset X_{j}$. However, low similarity values in some cases might not necessarily indicate that $u_{i}$ and $u_{j}$ have dissimilar preferences, but rather that $u_{i}$ might possibly be a cold user and his/her (still scarce) preferences would not be sufficient to ascertain how similar $u_{i}$ and $u_{j}$ are. This motivated us to introduce the multi-view fusion method based on weighted uninorm functions presented in this paper.

Example 3. Let $X=\left\{x_{1}, x_{2}, \ldots, x_{99}, x_{100}\right\}$ be a set of 100 items in a CFRS, and $u_{1}, u_{2} \in U$ two users in the system. Assume each user marked the following items as favorite, $F_{1}=\left\{x_{6}, x_{11}, x_{17}, x_{29}, x_{86}\right\}$ and $F_{2}=\left\{x_{4}, x_{6}, x_{29}, x_{54}, x_{86}, x_{90}\right\}$. The similarity degree between their preferences, $\operatorname{sim}_{f}^{12}$, is then calculated based on Eq. (7) as:

$$
\operatorname{sim}_{f}^{12}=\sqrt{\frac{\left|\left\{x_{6}, x_{29}, x_{86}\right\}\right|}{\left|\left\{x_{4}, x_{6}, x_{11}, x_{17}, x_{29}, x_{54}, x_{86}, x_{90}\right\}\right|}}=0.612
$$

\subsubsection{Preferences based on Explicit Rating Data}

We now describe how to calculate the preference-based similarity between explicit ratings. These generally include ratings expressed as numerical values in a finite scale (e.g. a 5-point scale) or a continuous interval (e.g. any value between 0 and 100). It is also possible to consider categorical, ordered rating scales, such as "Very Poor", "Poor", "Average", "Good", "Very Good", by mapping each category into a rating value in a finite, numerical scale, provided a lexicographical ordering exists. Without losing generality, the Cosine Coefficient on commonly rated items (which returns a similarity value in the unit interval), is adopted in this paper to calculate the preference similarity between the explicit ratings of two users. We refer the interested reader to [43] for a comprehensive overview of similarity measures between explicit ratings.

Let $r_{i}^{t} \in\left[r^{-}, r^{+}\right]$be an explicit rating by user $u_{i}$ on item $x_{t}$, expressed in a bounded rating domain $\left[r^{-}, r^{+}\right]$defined a priori, and let $\hat{r}_{i}^{t} \in[0,1]$ be the result of normalizing $r_{i}^{t}$ to the unit interval, i.e. $\hat{r}_{i}^{t}=\left(r_{i}^{t}-r^{-}\right) /\left(r^{+}-r^{-}\right)$. Denote by $X_{i}, X_{j} \subset X$ the subsets of items rated by $u_{i}$ and $u_{j}$, respectively. Also, let $R_{i}, R_{j}$ be two vectors containing all the actual ratings provided by $u_{i}$ and $u_{j}$, respectively. The cosine-based similarity between $u_{i}$ and $u_{j}$ in an explicit rating domain, $\operatorname{sim}_{f}^{i j} \in[0,1]$, is then calculated as follows:

$$
\operatorname{sim}_{f}^{i j}=\operatorname{sim}\left(R_{i}, R_{j}\right)=\frac{\sum_{t \in x_{i} \cap x_{j}} \hat{r}_{i}^{t} \cdot \hat{r}_{j}^{t}}{\sqrt{\sum_{t \in x_{i} \cap x_{j}}\left(\hat{r}_{i}^{t}\right)^{2}} \cdot \sqrt{\sum_{t \in x_{i} \cap x_{j}}\left(\hat{r}_{j}^{t}\right)^{2}}}
$$




\subsection{Similarity between User Profiles}

User profile information is a common, but frequently under utilized feature in the recommendation processes undertaken by most CFRS. In most CFRS-based platforms in the Internet, two types of features or information fields can be generally distinguished in a user profile:

1. Bounded information: It refers to fields of information whose possible values are bounded to a closed and finite set of options (e.g. by GUI elements such as check boxes, radio buttons, pull-down menus, etc). This restriction in possible values for a bounded fields makes coincidences between users typically more usual than in non-bounded information, particularly when the range of options to choose from is smaller.

2. Non-bounded information: It refers to fields of information whose value is freely introduced by the user, e.g. through text box GUI elements. Intuitively, since the content of these fields is less restricted than that of bounded information fields, coincidences between users in these fields are relatively more unusual.

In this work, we consider the distinct nature of these two types of information fields as a key aspect in measuring similarity between two user profiles, because coincidences in non-bounded information fields are normally more meaningful than coincidences in bounded information fields.

Let $C$ be the total number of occurrences for any elements of non-bounded profile information between two users. Unsurprisingly, due to the nature of this information, it is not frequent that $C>0$, i.e. the users share non-bounded information in common. Therefore, in most cases when $C=0$ the aggregation should be focused on combining bounded information solely to avoid downwards biased aggregation results. However, when $C>0$ the resulting profile similarity should be reinforced upwards, since rare occurrences must be regarded as indicators of two users with notable features in common among their profiles. We define an OWA-based aggregation process that reflects this rationale as follows: coincidences (similarity) between bounded profile features are used as the inputs to the OWA function, for each pair of users. Regarding non-bounded information, its number of common occurrences $C$ between two users is used to determine the OWA weighting vector $W$, such that:

1. It enables an optimistic aggregation attitude for non-zero values of $C$, such that the higher $C$, the more optimistic the resulting operator used and the closer the output is to the maximum, favoring the highest aggregation inputs.

2. It always assigns non-zero weights, i.e. all bounded information fields are taken into account to some degree, irrespective of the value of $C$.

Assume there are $n$ bounded information fields or features, $p_{1}, \ldots, p_{n}$. Given a feature $p_{z}$ (e.g. language/s spoken by a user) the similarity degree $\operatorname{sim}_{p_{z}}^{i j}$ between two users according to $p_{z}$ must be firstly calculated. This can be done by using a similarity measure based on the Jaccard index over the two subsets of values in $p_{z}$ selected by users $u_{i}$ and $u_{j}$ or, alternatively, by using fuzzy set theory $[15,44]$ and calculating a (fuzzy) degree $\mu_{s i m}\left(p_{z}^{i j}\right)$ of similarity between $u_{i}$ and $u_{j}$ in $p_{z}$ (e.g. number of commonly spoken languages). The second option becomes more appropriate if the number of possible values to choose from $p_{z}$ is reasonably large. Similarities on bounded features are subsequently aggregated:

$$
\operatorname{sim}_{p}^{i j}=O W A_{W}\left(\operatorname{sim}_{p_{1}}^{i j}, \ldots, \operatorname{sim}_{p_{n}}^{i j}\right)
$$

The number of occurrences in non-bounded information, $C$, is used to calculate the OWA weights based on an increasing function $\phi=0.5^{\frac{1}{C+1}}, \phi \in[0.5,1]$ of $C$ of $C$ whose effect is to reinforce the weights of larger input values and penalize the weights of lower input values, as $C$ increases.

$$
w_{z}^{\prime}=1+\frac{(z-1)(1-2 \phi)}{n-1}
$$

Weights $w_{z}^{\prime} \in[0,1]$ are subsequently normalized:

$$
w_{z}=\frac{w_{z}^{\prime}}{\sum_{z} w_{z}^{\prime}}
$$

Importantly, $w_{1}^{\prime}=1, \forall C$, i.e. the largest element always has a weight of 1 before normalizing. If $C=0$, then $\phi=0.5$ and the fractional term in Eq. (11) becomes zero, thus $w_{1}^{\prime}=1, \forall z \in\{1, \ldots, n\}$, in other words, the OWA aggregation 
Table 1: Uninorm-based computation of $\operatorname{sim}^{i j}$

\begin{tabular}{|c||c|c|c|}
\hline$\left(u_{i}, u_{j}\right)$ & $\operatorname{sim}_{f}^{i j}$ & $\operatorname{sim}_{p}^{i j}$ & $\operatorname{sim}^{i j}$ \\
\hline$\left(u_{1}, u_{2}\right)$ & 0.612 & 0.409 & $\mathbf{0 . 5 3 2}$ \\
\hline$\left(u_{1}, u_{3}\right)$ & 0.38 & 0.415 & $\mathbf{0 . 3 0 3}$ \\
\hline$\left(u_{1}, u_{4}\right)$ & 0.5 & 0.437 & $\mathbf{0 . 4 3 7}$ \\
\hline$\left(u_{1}, u_{5}\right)$ & 0.675 & 0.58 & $\mathbf{0 . 7 4 1}$ \\
\hline
\end{tabular}

behaves as an arithmetic average of bounded features (with neutral attitude) when two users have no coincidences in non-bounded profile information. Conversely, if $C>0$, the fractional term in Eq. (11) takes a negative value in $[-1,0]$ that penalizes the overall $w_{z}^{\prime}$ : the lower the value to aggregate, the more penalizing is applied to its associated weight. Likewise, the larger $C$, the less evenly distributed weights in $W$ will be, with more accentuated differences between highest and lowest importance weights, resulting in a more optimistic aggregation. Section 4.2 illustrates the application of the proposed OWA aggregation method in the case study.

\subsection{Multi-View Similarity Aggregation}

Once calculated the two views of similarity between users, in terms of their preferences and their user profile, both views are fused to obtain a global degree of similarity between the target user $u_{i}$ and the rest of users in the system. As pointed out in the introduction, a central requirement in this multi-view fusion procedure consists in emphasizing situations when two users simultaneously present a high (resp. low) similarity degree in both their preference and profile views. Thus, we utilize a uninorm aggregation function to obtain the global similarity $\operatorname{sim}^{i j} \in$ $[0,1]$ between $u_{i}$ and $u_{j}$, as the full reinforcement property exhibited by uninorm functions makes them suitable to meet this requirement:

$$
\operatorname{sim}^{i j}=\mathcal{U}\left(\operatorname{sim}_{f}^{i j}, \operatorname{sim}_{p}^{i j}\right)
$$

with $\mathcal{U}$ a uninorm function in $[0,1]^{2}$, e.g. the cross-ratio uninorm (Eq. (3)), with $g=0.5$. If $\operatorname{sim}_{f}^{i j}>g$ and $\operatorname{sim}_{p}^{i j}>g$ then the aggregated similarity $\operatorname{sim}^{i j}$ between $u_{i}$ and $u_{j}$ is even higher (upward reinforcement) to reflect that both users are very similar. Conversely, if $\operatorname{sim}_{f}^{i j}<g$ and $\operatorname{sim}_{p}^{i j}<g$ then $\operatorname{sim}^{i j}$ becomes lower (downward reinforcement) to reflect that the two users do not present similar traits to each other. Finally, if $\operatorname{sim}_{f}^{i j}<g<\operatorname{sim}_{p}^{i j}$ then the resulting $\operatorname{sim}^{i j}$ lies in between both similarity views (averaging behavior). Different families of uninorm operators [41], could be flexibly utilized depending on a predominantly disjunctive or conjunctive behavior being required in the aggregation. The higher $g$ in the function $\mathcal{U}$ chosen, the more predominantly conjunctive its behavior is, and vice versa. In other words, the higher $g$, the more restrictive the recommender algorithm becomes in the identification of similar users.

Example 4. Table 1 shows the preference, profile and overall similarity values computed between user $u_{1}$ and four more users, $u_{2}, u_{3}, u_{4}, u_{5}$. It can be noticed the upward reinforcement $\left(\operatorname{sim}^{15}\right)$, downward reinforcement $\left(\operatorname{sim}^{13}\right)$, and averaging behavior $\left(\operatorname{sim}^{12}\right)$ on the aggregated value, depending on values to aggregate being higher or lower than 0.5. A neutral similarity degree can be also observed in $\operatorname{sim}^{14}$.

\subsection{Managing Cold Users}

The cold-start problem, which appears when the number of available ratings is relatively small [29], is a common drawback frequently found in CFRS, whose rationale has been extensively studied in related literature [8]. In this subsection we introduce a method based on fuzzy set theory to alleviate the "new user" cold-start problem, and we describe its integration with the proposed multi-view aggregation framework for collaborative filtering. We herein focus on alleviating the cold-start problem for new users [30], i.e. users who rated a small number of items in the system.

Remark 2. The transition of a user from being cold (new) to becoming a "well-established" user across the time is smooth, in the sense that users normally construct a consolidated rating history gradually, rather than abruptly.

\footnotetext{
${ }^{1}$ The term "well-established" is used herein to refer to those users with sufficient ratings not to be deemed as cold users.
} 
Intuitively, this means that the boundary between cold and well-established user is not crisp, but rather vague and imprecise in nature. Based on this, our proposal herein makes use of fuzzy set theory to give an answer to the question "When does the number of user ratings become large enough to rely on ratings to produce recommendations?". Likewise, we aim at modeling the cases when the user is neither clearly cold nor well-established, but instead transiting between both (partly cold).

Within the context of the proposed multi-view CFRS model, when a user $u_{i}$ has marked a small (or even null) number of items as favorite, its preference-based similarity with respect to another well-established user $u_{j}$ might bias the real overall similarity with $u_{j}$ in some cases, as the existing preferential information or rating history is still not sufficient to conclude that both users do not have similar features with each other. Consider for example an implicit rating scenario, in which there are two users $u_{1}, u_{2}$ with $\left|X_{1}\right|=2,\left|X_{2}\right|=24, \operatorname{sim}_{f}^{12}=0.05$ and $\operatorname{sim}_{p}^{12}=0.7$. Clearly, $u_{1}$ is a cold user who rated two items only, and the resulting similarity obtained by using a non-weighted uninorm function, $\operatorname{sim}^{12}=\mathcal{U}(0.05,0.7)$ is strongly biased downwards by $\operatorname{sim}_{f}^{12}$, even though both users have a highly similar profile. In order to avoid these situations and improve aggregation results under the pressence of cold users, our solution for the cold-start problem in the proposed multi-view CFRS model focuses on the use of a weighted uninorm function. This way, an importance degree can be assigned to each similarity view for a given pair of users, depending on one (or both) of them being a cold user.

The approach utilized to assign weights to the aggregation function $\mathcal{U}$ relies on fuzzy set theory [15], in order to properly capture the inherent vagueness behind the concepts of cold versus well-established user. Put another way, it is neither realistic nor trivial to establish a crisp boundary between a cold user and a well-established user in the system, in terms of his/her number of items rated, $\left|x_{i}\right|$. Let $C O L D$ be a fuzzy set defined on $\mathbb{R}^{+}$, with nonincreasing membership function $\mu_{C O L D}(x), x \in[0, \infty]$ (also expressed hereinafter as $\mu_{C}(y)$ for notation convenience). This fuzzy set is utilized to model the (imprecise) notion of cold user. The (semi-trapezoidal) fuzzy membership function associated has the form,

$$
\mu(y)=\left\{\begin{array}{cl}
1 & \text { if } y \leq \alpha \text { (cold), } \\
\frac{\beta-y}{\beta-\alpha} & \text { if } \alpha<y<\beta \text { (partly cold), } \\
0 & \text { if } y \geq \beta \text { (well-established). }
\end{array}\right.
$$

with $y \in \mathbb{R}^{+}$representing the number of items rated by a user. The values of two parameters $\alpha, \beta$ of the membership function allow for a better classification of users into cold, partly cold and well-established users, depending on the actual value of $y$ (which, in our context, corresponds to $\left|X_{i}\right|$ ). It is noteworthy that the distinction made here between three types of users has the sole purpose of assigning the most appropriate importance weights to each type of user during the aggregation of pairwise similarity views, as explained below, and the set of users is not divided into subgroups according to their type. Despite the values of $\alpha, \beta$ are normally fixed a priori in most applications, in a RS domain their specification is not compulsory: instead, they can be automatically adjusted based on the total number of items in the system $|X|$, i.e. $\alpha=f_{\alpha}(|X|), \beta=f_{\beta}(|X|)$, such that $f_{\alpha}(|X|)<f_{\beta}(|X|) \ll|X|$. The functions $f_{\alpha}$ and $f_{\beta}$ can simply be of the form $\tau \cdot|X|$ with $\tau \approx 0$ a positive value close to zero, i.e. $\alpha$ and $\beta$ are calculated as a (small) proportion of $|X|$. Cold users tend to be underrated or overrated by any CFRS that relies on user similarity based on commonly rated items solely, since the chances that such similarity is low or even null are higher, when compared against well-established users in the system. This may affect the computation of the overall similarity between two users when at least one of them is a cold user.

Our solution is different from other approaches to cope with the cold-start problem, in that it suits our overall proposal of multi-view intelligent aggregation-based CFRS model. More specifically, a weighted uninorm function $\mathcal{U}_{W}\left(\operatorname{sim}_{f}^{i j}, \operatorname{sim}_{p}^{i j}\right)$ is now defined to calculate $\operatorname{sim}{ }^{i j}$, with $W=\left[w_{f}, w_{p}\right]$ a weighting vector such that $w_{f}$ is the importance assigned to the preference-based similarity view, and $w_{p}$ is the importance assigned to the profile-based similarity view. The weighting vector is defined differently depending on the fuzzy classification of users $u_{i}$ and $u_{j}$ :

1. Both $u_{i}$ and $u_{j}$ are cold users: This occurs when the number of items rated by both users is lower than $\alpha$, thus $\mu_{C}\left(\left|X_{i}\right|\right)=\mu_{C}\left(\left|X_{j}\right|\right)=1$. Intuitively, the chances that two "new" users in the system have one commonly rated item are particularly low, therefore any $\operatorname{sim}_{f}^{i j}>0$ should be regarded as an indicator of two users with potentially similar interests. On the other hand, if $\operatorname{sim}_{f}^{i j}=0$ it is more sensible to rather rely on the user profile 


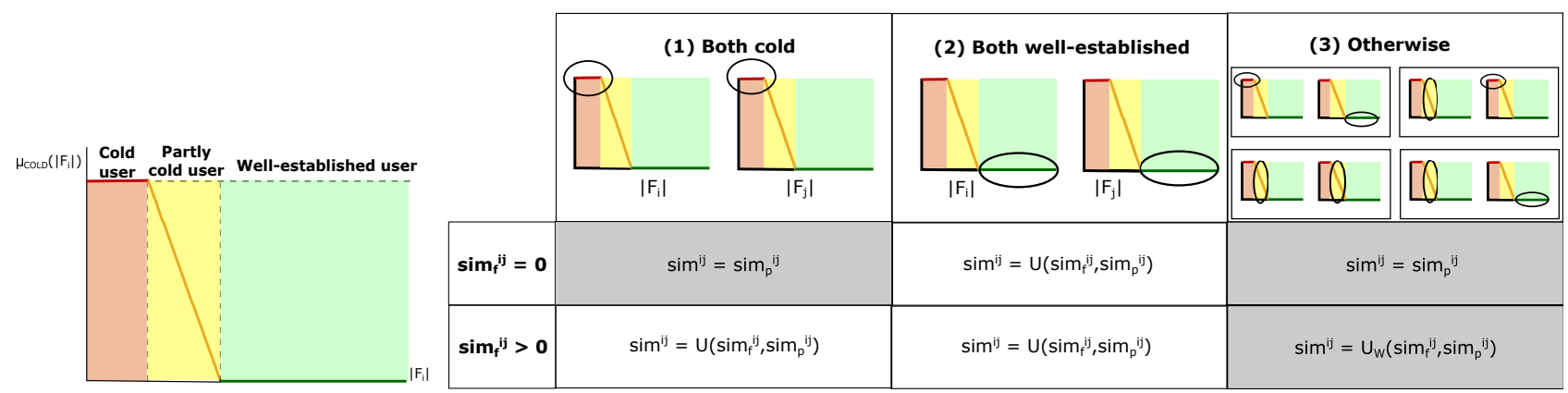

Figure 4: Fuzzy membership function COLD (left); behavior of the weighting and fusion of $\operatorname{sim}_{f}^{i j}$ and $\operatorname{sim}_{p}^{i j}$ into $\operatorname{sim}^{i j}$ (right).

similarity view. Thus, for two cold users we assign $w_{p}=1$ and,

$$
w_{f}= \begin{cases}0 & \text { if } \operatorname{sim}_{f}^{i j}=0 \\ 1 & \text { otherwise }\end{cases}
$$

2. Both $u_{i}$ and $u_{j}$ are well-established users: When the number of ratings made by $u_{i}$ and $u_{j}$ is large enough $\left(\mu_{C}\left(\left|X_{i}\right|\right)=\mu_{C}\left(\left|X_{j}\right|\right)=0\right)$ the CFRS can fully rely on both similarity views by using a classical uninorm aggregation function. This is equivalent to assigning a equal, maximum weight to both views. In other words, if $w_{f}=1$ and $w_{p}=1$, then the weighted uninorm acts as a classical, non-weighted uninorm function $\mathcal{U}$.

3. Neither (1) nor (2) hold: This occurs in the remaining cases, i.e. when (i) at least one of the two users is a partly cold user, or (ii) $u_{i}$ is cold user and $u_{j}$ is well-established (or vice versa). Two sub-cases are distinguished here. Firstly, when the two users do not have common ratings, $\operatorname{sim}_{f}^{i j}=0$, only the profile information is considered to avoid missing potentially affine users (for the same reason as described above for two cold users). Secondly, if $\operatorname{sim}_{f}^{i j}>0$, similarity views are weighted under the principle that if two users are partly cold to a similar degree, common preferences among them are regarded as meaningful as common profile features. Contrarily, if one of the users has considerably more established preferences than the other (which means that the letter has a low number of preferences/ratings), common preferences should be regarded as less meaningful than common profile features. To express this idea in terms of membership degree to $C O L D$, the bigger the difference between $\mu_{C}\left(\left|X_{i}\right|\right)$ and $\mu_{C}\left(\left|X_{j}\right|\right)$, the less relevant the preference view is deemed, compared to the profile view. This behavior can be captured by using a weighting scheme in which $w_{p}=1$ and:

$$
w_{f}= \begin{cases}0 & \text { if } \operatorname{sim}_{f}^{i j}=0 \\ 1-\frac{\left|\mu_{C}\left(\left|X_{i}\right|\right)-\mu_{C}\left(\left|X_{j}\right|\right)\right|}{2} & \text { otherwise }\end{cases}
$$

with $w_{f} \in[0.5,1]$. In the particular case that $\mu_{C}\left(\left|X_{i}\right|\right)=\mu_{C}\left(\left|X_{j}\right|\right)$, we then have $w_{f}=0$, which occurs when $u_{i}, u_{j}$ are both partly cold to an equal extent, thus weighing their preference similarity the same way as in the case of two cold users described above. The opposite case occurs when a user is cold and the other is well-established, i.e. $\mu_{C}\left(\left|X_{i}\right|\right)=0$ and $\mu_{C}\left(\left|X_{j}\right|\right)=1$, or vice versa, which leads to $w_{f}=0.5$, i.e. the importance of the preference view is half less important than the importance of the profile view.

Figure 4 summarises in a simplified manner the behavior of the multi-view similarity aggregation in pairs of cold, partly cold and well-established users.

Example 5. Consider a CFRS model with $|X|=1000, \alpha=5, \beta=10$, and three users $u_{1}, u_{2}, u_{3} \subset U$. Based on the values of $\alpha, \beta$, suppose that $(i) u_{1}$ is a cold user with $\left|F_{1}\right|=1$, (ii) $u_{2}$ is a partly cold user with $\left|F_{2}\right|=8$, and (iii) $u_{3}$ is a well-established user with $\left|F_{3}\right|=17$. Suppose all three users have a highly similar profile to each other, with $\operatorname{sim}_{p}^{12}=\operatorname{sim}_{p}^{13}=\operatorname{sim}_{p}^{23}=0.7$, but they all have only one commonly rated item with each other. The resulting preference-based similarities are, $\operatorname{sim}_{f}^{12}=0.125$, $\operatorname{sim}_{f}^{13}=0.059$ and $\operatorname{sim}_{f}^{23}=0.042$. By applying the weighting scheme to manage cold users (see Figure 4) based on a cross ratio weighted uninorm and the uninorm weighting operator in Eq. (4), we have that for all three different pairs of users, $\operatorname{sim}^{i j}=\mathcal{U}_{W}\left(\operatorname{sim}_{f}^{i j}, \operatorname{sim}_{p}^{i j}\right)$ is calculated as follows: 
- For $u_{1}, u_{2}$, we have $w_{f}=0.7$ and $w_{p}=1$. By applying the uninorm weighting operator, we have $\mathcal{U}_{W}(0.125,0.7)=$ $\mathcal{U}(0.2375,0.7)$, i.e. the preference view similarity is slightly increased after penalizing its weight.

- For $u_{1}$ and $u_{3}$ the resulting weights of views are $w_{f}=0.5$ and $w_{p}=1$, due to one of them being cold and the other well-established, and $\mathcal{U}_{W}(0.059,0.7)=\mathcal{U}(0.2795,0.7)$. Here, the influence of the weighting scheme is even stronger, even though the initial preference view had an almost null similarity degree.

- For $u_{2}$ and $u_{3}$, we have $w_{f}=0.8$ and $w_{p}=1$, since none of the users is totally deemed as a cold user, the weight $w_{f}$ is kept closer to $w_{p}$, and thus $\mathcal{U}_{W}(0.042,0.7)=\mathcal{U}(0.1336,0.7)$.

\subsection{Identifying Neighbors and Delivering Recommendations}

Once computed $\operatorname{sim}^{i j}$ for a target user $u_{i}$ and every $u_{j}, j \neq i$, a $k N N$ algorithm is applied to determine the $k$ most similar users to $u_{i}$. Let $\sigma(\cdot)$ be a permutation function over the index set $\{1, \ldots, m\}$. Let $U_{\sigma}=\left\{u_{\sigma(1)}, \ldots, u_{\sigma(m-1)}\right\}$, with $u_{\sigma(j)} \in U \backslash\left\{u_{i}\right\}$, be the set formed by all users except for $u_{i}$, ordered by decreasing order of similarity ${ }^{2}, \operatorname{sim}^{i, \sigma(j)}$, such that $\left(\operatorname{sim}^{i, \sigma(j)}\right) \geq\left(\operatorname{sim}^{i, \sigma(j+1)}\right), \forall j \in\{1, \ldots, m-2\}$. Given $k \ll m$, the $k$ neighbor users of $u_{i}$ are determined as the top- $k$ elements in $U_{\sigma}$, i.e. $U_{\sigma}^{k}=\left\{u_{\sigma(1)}, \ldots, u_{\sigma(k)}\right\} \subseteq U_{\sigma}$.

Subsequently, the preferences of neighbor users are utilized to predict the degree to which items not rated by $u_{i}$ yet, $x_{t} \in X \backslash X_{i}$, might be of interest to her/him. Unlike users' preferences, which are expressed as unary ratings $r_{i}^{t}$ in $\{0,1\}$, predicted ratings can lie anywhere in the $[0,1]$ interval. The prediction value for user $u_{i}$ over item $x_{t}$, denoted by $p\left(u_{i}, x_{t}\right) \in[0,1]$, is calculated as a weighted average of all neighbors' preferences over $x_{t}$, as follows:

$$
p\left(u_{i}, x_{t}\right)=\frac{\sum_{j=1}^{k} \operatorname{sim}^{i, \sigma(j)} \cdot r_{\sigma(j)}^{t}}{\sum_{j=1}^{k} \operatorname{sim}^{i, \sigma(j)}}
$$

$r_{\sigma(j)}^{t} \in\{0,1\}$ indicates whether $x_{t}$ has been marked as favorite by $u_{\sigma(j)}$ or not:

$$
r_{\sigma(j)}^{t}= \begin{cases}1 & \text { if } x_{t} \in X_{j} \\ 0 & \text { otherwise }\end{cases}
$$

As we can observe, similarity degrees $\operatorname{sim}^{i, \sigma(j)}$ play the role of importance weights in the computations of $p\left(u_{i}, x_{t}\right)$, such that preferences of the most similar users to $u_{i}$ attain higher importance in the calculation of the predicted rating for $x_{t}$.

Finally, a recommendation list $\Lambda_{i}=\left\{\lambda_{1}, \ldots, \lambda_{h}\right\}$ of size $h \in \mathbb{N}, h \ll l$, is generated and delivered to $u_{i}$. Such a list contains $h$ recommendations, given by those $h$ items $\lambda_{k}=x_{t} \in I$ with the highest values for $p\left(u_{i}, x_{t}\right)$. Thus, the target user receives a list of unrated items $\lambda_{k}$, ordered by decreasing value of $p\left(u_{i}, x_{t}\right)$.

\section{Case Study}

In this section we illustrate and validate the practical application of our proposed methodology. Although the case study presented is mainly orientated towards an application in the Urban Resilience domain (Sections 4.1, 4.2 and 4.3) for which a prototypical recommender model was intially conceived [12], we also illustrate the applicability of the proposed approach in different domains, such as movie recommendation via a profile-based extension of the popular Movielens dataset (Section 4.4).

The data has been obtained from the HARMONISE platform. Users to the platform are required to register at the first instance of use. Registration captures important profile information including the users area/s of expertise, lines of investigation and areas of interest (such as planning and design, public buildings infrastructure), as well as language/s and resilience groups/communites they belong to or they are interested in. Using these data we will specifically highlight how the multi-view approach using the weighted uninorm based on profile and preference similarity has an impact on prediction accuracy. Furthermore, we present how the weighting scheme in Section III can address the issue

\footnotetext{
${ }^{2}$ For the sake of clarity in notation, a comma is introduced in the similarity superscript in specific cases to avoid confusion, as occurs e.g. with $\operatorname{sim}^{i, \sigma(j)}$.
} 
of cold users. The steps involved with the CFRS procedure implemented in this Case Study are based on those shown in Algorithm 1. These include the combination of both profile and preference based similarity (by taking account of cold users), the identification of nearest neighbors and the predicted ranking of recommendations for individual users.

A comprehensive use case scenario of use of the HARMONISE platform functionalities, including content recommendation, is presented in [19].

\subsection{User and Urban Resilience Resource Data}

The data applied in this Case Study has been extracted from the HARMONISE platform database and consists of user profile and favorite information alongside the set of urban resilience resources available. A summary of this data utilized for the evaluation of the CFRS algorithm is described as follows. A set of 3.5k users in total was obtained. These 3k users are distributed into the three types introduced in Section 3.4, i.e. depending on their level of "establishment" measured in terms of favorite items marked.

- Well-established users $(\approx 1.5 k, 42 \%)$ : marked 10 or more items as favorite.

- Partly cold users $(\approx 1 k, 29 \%)$ : marked 5 of more, but less than 10 items as favorite.

- Cold users $(\approx 1 k, 29 \%)$ : marked less than 5 items (or none) as favorite.

As our proposed approach makes use of profile features in the user similarity calculation, user profile information captured for users was extracted. These consisted of one non-bounded field (lines of investigation) and three bounded fields described as follows:

- Languages: Users speak one or more among a total of 14 different languages.

- Groups: Users in the system belong to at least one out of three different groups.

- Topics: Users belong to one or more of the 10 different topics or areas of expertise defined within the urban resilience life cycle.

The HARMONISE platform contains a set of over $2.8 \mathrm{~K}$ urban resilience resources ranging from documentation of specifications through to urban planning tools. Over 31k ratings (favorite markings) for this set of resources were gathered from the $3.5 \mathrm{k}$ users.

\subsection{Calculating Profile Similarity in the HARMONISE platform}

User profile similarity is calculated according to a number of relevant profile information features, straddling three bounded information fields and one non-bounded field which are stored on the HARMONISE database:

1. Groups (bounded): This feature refers to the different urban resilience communities each user belongs to, $G_{i} \subseteq$ $G$, being $G$ the set of all existing groups in the system.

2. Topics (bounded): Topic/s of interest for the user, $T_{i} \subseteq T$, being $T$ the set of all existing topics in the system. There is a pre-defined set of 13 different topics, being all of them related to urban resilience (e.g. "natural disaster", "man-made accident", etc.).

3. Languages (bounded): Language/s spoken by the user, $L_{i} \subseteq L$, with $L$ the set of all available languages.

4. Lines of Investigation (non-bounded): Lines of investigation in which the user is interested. Unlike topics, there are no pre-defined lines of investigation in the system, therefore the user freely introduces type the line/s he/she works on (e.g. "decision support systems", "robotics", "management") when configuring the user profile.

By exhaustively analyzing the users' profiles database we noticed that finding a line of investigation between any two users was relatively infrequent compared to finding (pairs of) users with common groups, topics or languages. Nonetheless, by using the proposed OWA weighting approach described in Section III, particular cases when two users have one or more lines of investigation in common can be taken into consideration, by positively reinforcing the overall user profile similarity obtained upon an appropriate weighting of the three bounded features to be aggregated. Thus, the group, topic and language-based similarities $\operatorname{sim}_{G}^{i j}, \operatorname{sim}_{T}^{i j}$ and $\operatorname{sim}_{L}^{i j}$ are aggregated by instantiating the OWA operator introduced in Eq.(10) into: 

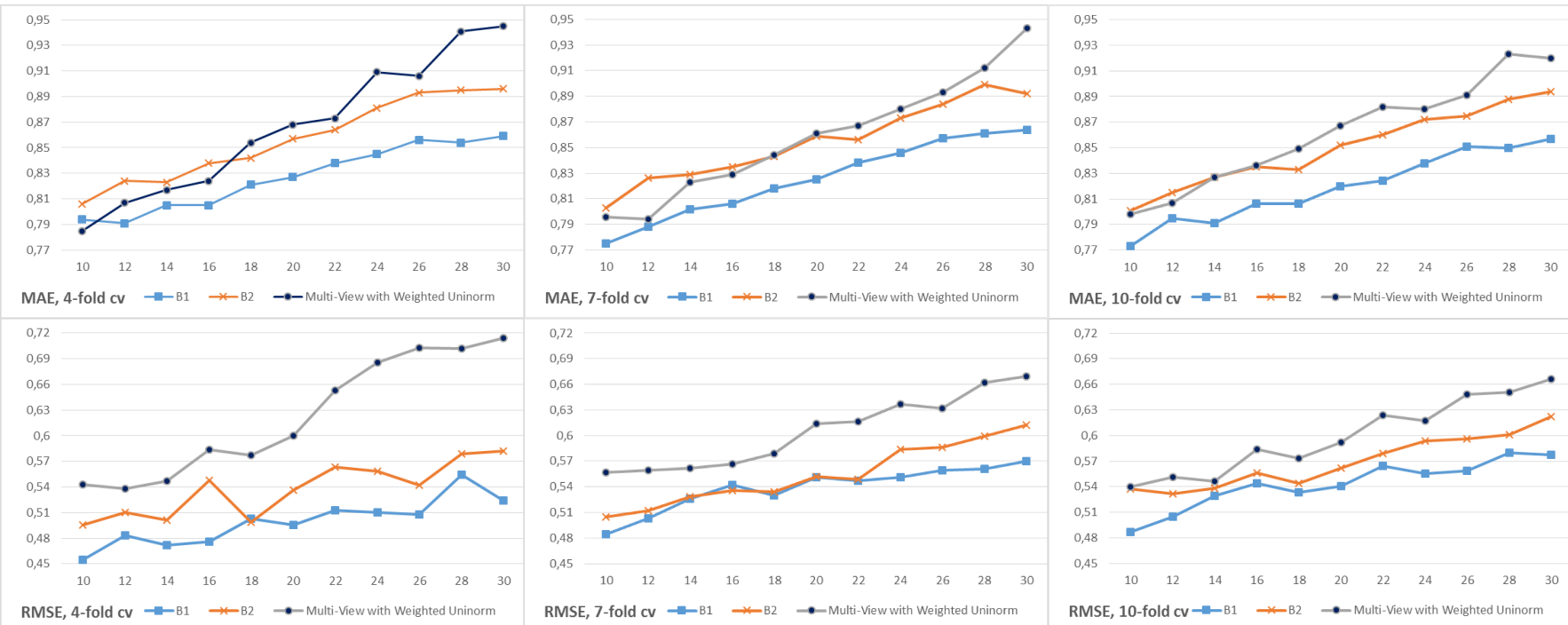

Figure 5: Comparison between the prediction accuracy of the proposed multi-view fusion approach against two baselines: (B1) based on user preferences (ratings) only, and (B2) without managing cold users (non-weighted uninorm), across various KNN selections and numbers of folds

$$
\operatorname{sim}_{p}^{i j}=O W A_{W}\left(\operatorname{sim}_{G}^{i j}, \operatorname{sim}_{T}^{i j}, \operatorname{sim}_{L}^{i j}\right)
$$

with the following non-normalized weights:

$$
W^{\prime}=\left(1, \frac{3-2 \phi}{2}, 2-2 \phi\right)
$$

It is clear from Eq.(20) that the more non-bounded information two user profiles have in common ( $\phi$ closer to one), the more importance is assigned to the most similar profile features.

\subsection{Evaluation in the HARMONISE platform}

We performed a comparative analysis to demonstrate the effectiveness of the multi-view approach using a weighted uninorm function, consisting of the cross ratio uninorm with neutral element $g=0.5$, and the uninorm weighting operator introduced in Eq. (4). For the identification of cold and partly cold users, we have considered a fuzzy set with $\alpha$ and $\beta$ equal to 5 and 10, respectively ${ }^{3}$, both of which are below the $1 \%$ of the total number of items used in the evaluation.

The experimental evaluation consists of an offline evaluation and an online evaluation. The offline evaluation is aimed at comparing the proposed multi-view approach using the OWA and weighted uninorm with two baseline methods, characterised by: (B1) using preference similarity only, and (B2) calculating multi-view similarity without weighting in the uninorm aggregation (i.e. cross ratio aggregation on both views of similarity, without weighting them). These evaluations were performed through measuring the predictive accuracy of the three approaches described

\footnotetext{
${ }^{3}$ Based on a fuzzy relaxation of the MovieLens database settings, which consider 15 as the threshold number of ratings for an user to receive meaningful recommendations.
} 
Table 2: \% of ratings marked as favorite via a recommendation list.

\begin{tabular}{|c|c|c|c|c|c|c|}
\hline 1 & 2 & 3 & 4 & 5 & 6 & 7 \\
\hline $80.71 \%$ & $74.96 \%$ & $75.31 \%$ & $85.16 \%$ & $84.49 \%$ & $68.60 \%$ & $77.28 \%$ \\
\hline
\end{tabular}

above. To do this, we utilize the Mean Absolute Error (MAE) and the Root Mean Squared Error (RMSE), as prediction accuracy metrics:

$$
\begin{gathered}
\mathcal{P}_{M A E}=1-\frac{1}{|R|} \sum_{r_{i}^{t} \in R}\left|r_{i}^{t}-p\left(u_{i}, x_{t}\right)\right| \\
\mathcal{P}_{\text {RMSE }}=1-\sqrt{\frac{\sum_{r_{i}^{t} \in R}\left(r_{i}^{t}-p\left(u_{i}, x_{t}\right)\right)^{2}}{|R|}}
\end{gathered}
$$

with $R \subset U \times X$ a subset of user-item ratings used as the test subset for evaluation, and $r_{i}^{t} \in\{0,1\}$ as in Eq. (18).

We use a cross validation to obtain an average value of the prediction metric, with three different settings in the number of folds: 4-fold, 7-fold and 10-fold. For each of these cross validation settings, the test fold $R$ has been selected based on a Simple Random Sampling (SRS) over $U \times X$. The results obtained for different settings of the neighborhood size $k$ and number of folds, with a recommendation list size $h=5$, are summarized in Figure 5 .

In general, as $k$ increases, all three approaches improve their accuracy, as more user information is taken into account for making predictions. However, it can be observed how the proposed multi-view with weighted uninorm approach outperforms the preference only similarity (B1) and multi-view without uninorm weight (B2) as the neighborhood size increases. This is because, when using the multi-view method that incorporates user profile information in similarity computation, potentially similar users which are left out of the neighborhood with the baseline method, are incorporated in the neighborhood. Furthermore, the use of a weighted uninorm to aggregate both views further increases the accuracy, partly due to improving predictions for cold and partly cold users, by relying more on user information obtained from their profile. The weighting of similarities involving at least one cold users sometimes affects the overall similarity among two users and, in turn, it may determine whether a user $u_{j}$ might be considered or not as part of $u_{i}$ 's neighborhood: ignoring cold users might in some cases lead to leaving out potentially relevant neighbors, and adopting other least affine users as neighbors instead, thus affecting the final recommender performance. This becomes noticeable in Figure 5 as the neighborhood size increases, but for a low $k$ the scheme to managing cold users does not add apparent benefits over the overall OWA and Uninorm-based scheme (which makes sense because the most highly similar neighbors to a target user normally are well-established users, according to the proposed fuzzy categorisation of users). Finally, the difference in accuracy between our proposed method and (B1) is more apparent when using the RMSE as the prediction accuracy metric, which indicates that the presence of larger error rates is more frequent in (B1).

Additionally, an online standalone evaluation has been conducted to measure the direct impact of the CFRS model on the end user, in other words, we measure how the users' reactions are with respect to the recommendations provided. To do this, we examine a sample of items marked as favorite by users, $R_{1}=\left\{r_{i}^{t}: r_{i}^{t}=1\right\} \subseteq R, R \subset U \times X$ and determine which ones have been accessed and marked as favorite via a recommendation list provided to the user (contrary to e.g. accessing the item via an ordinary search). This information is used to estimated an average percentage of items in recommendation lists that are viewed and marked as favorite by the user, thus providing an estimate of the level of novelty and serendipity [29]. We divide the overall subset $R_{1}$ of favorite ratings into 7 samples via SRS, and calculate the average \% of recommended items marked as favorite as shown in Table 2 . The significantly high percentages show that users of the HARMONISE platform largely rely on recommendations provided by the proposed multi-view fuzzy information fusion method.

\subsection{Application Example in Movie Recommendation}

In order to demonstrate the applicability of the proposed fusion approach in a more general array of recommender domains, in this subsection we explore its applications to the problem of recommending movies, predicated on explicit 


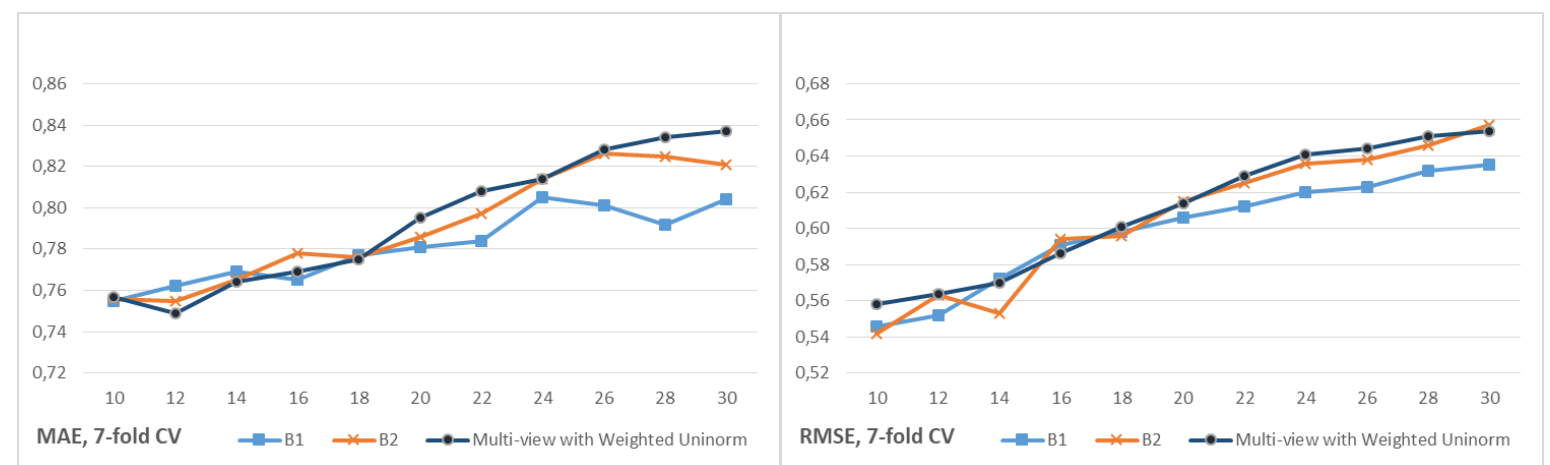

Figure 6: Comparison between the prediction accuracy of the proposed multi-view fusion approach against two baselines: (B1) based on user preferences (ratings) only, and (B2) without managing cold users (non-weighted uninorm), across various KNN selections in movie recommendation

ratings and relevant user profile information. To do this, we have extended the well-known "MovieLens 100k" dataset ${ }^{4}$, so as to incorporate both preference data (based on explicit numerical ratings) and comprehensive user profile data for $1 \mathrm{k}$ users, part of which has been generated synthetically:

- User preferences: They consist of explicit, numerical ratings on movies, on a 1-5 numerical scale.

- User profile: Each user profile consists of three bounded fields and one non-bounded field. Demographic information contained in the existing Movielens database is extended by adding synthetic data pertaining two additional profile fields.

- Occupation: This field relates to the occupation(s) of the user. We deem the user occupation as a nonbounded field, due to the wider variety of possible values for it.

- Age group: Based on the age attribute from the base dataset, we create a bounded profile field for different age groups, e.g. $<18,18-25,25-35,35-45,45-65$, and $>65$.

- Languages: Language(s) understood by the user, this bounded profile field has been generated synthetically and integrated with the base Movielens 100k dataset.

- Favorite genres: The Movielens database contains genre information associated to movies. However, we are interested in modeling the favorite genres of the user as part of her/his profile, hence we add a bounded profile field with synthetically generated data, in which a user can have at least one favorite genre out of the 19 existing ones.

The same instance of OWA operator from Eqs. (19) and (20) is used here to aggregate partial profile similarities. To better fit the nature of the Movielens rating data, in which every user has at least 20 ratings, we set $\alpha=25$ and $\beta=30$. Furthermore, the recommendation list size here is $h=10$. A longer recommendation list implies that there would be larger differences between the predicted ratings for items at the top and bottom of such a list. Moreover, a 7-fold cross validation with SRS for sampling a subset of test users are considered. We evaluate the precision of recommendations (see Eqs. (21) and (22)) against baseline versions B1 and B2, as described earlier on in this section. Results are summarized in Figure 6. On the one hand, the CFRS with the proposed multi-view fusion approach tends to outperform B1 as the neighborhood size $k$ increases. On the other hand, in this case there are no notable differences between the performance of our proposal and B2. We argue that this is because fewer users are identified as partly cold and cold (users in Movielens generally have a reasonable number of ratings) and, accordingly, the uninorm aggregation of similarity views behaves more similarly in both versions.

\footnotetext{
${ }^{4}$ Original Movielens data available at: https://grouplens.org/datasets/movielens/100k/
} 


\section{Conclusions and Future Work}

In this paper we have presented our proposed multi-view user information fusion approach in CFRS using weighted uninorms. As systems continue to improve in terms of the quality and quantity of feature data available to the RS, it will become imperative to fully utilize this rich data resource for tailored recommendations. Our approach has taken into consideration such rich feature information in terms of user profiles and preferences to enhance the tailoring of content to users in the Urban Resilience domain. These features are often under utilized in current RS approaches. We have aggregated these multiple features using OWA operators to dynamically assign weights to feature elements, capture the meaningfulness of coincidences between users in different aspects of their profile, and fuse together different features based on their similarity. As demonstrated in the Case Study, integration of multiple relevant features is promising in delivering accurate domain-specific recommendations. The use of uninorm functions in the similarity calculation process is another contribution of this work. These functions have provided the capability to apply reinforcement when aggregating features of similarity, in this case, preference-based and profile-based similarity. Furthermore, we have addressed the "new user" cold start problem through the integration of uninorm functions with a weighted scheme based on fuzzy set modeling, which effectively captures the imprecise notion of cold user. Our proposed model has been demonstrated in the domain of Urban Resilience to improve the filtering and predicting relevant content to users in this domain, as well as in the problem of recommending movies based on both user preferences and user profile information. The key concepts outlined in the paper could be extended to other problem domains suffering from similar issues.

In future work we will extend the exploration of integrating more user features in the context of CFRS, such as inter-user trust. Furthermore, we will expand our work on modeling weights and consider other important user preferences (e.g. navigation-based inference of implicit preferences) to take into consideration their impact on the RS process. Finally, we aim at investigating the "new item" cold start problem, and the jointly occurrence of cold users and items, by extending the proposed fuzzy set-based method.

\section{Acknowledgments}

The work was supported by the HARMONISE project (A Holistic Approach to Resilience and Systematic Actions to make Large Scale UrbaN Built Infrastructure Secure, www.harmonise.eu) co-funded by the European Commission within the Seventh Research Framework Programme (20072013) Under Grant Agreement number 312013.

\section{References}

[1] F. Ricci, L. Rokach, and B. Shapira, Introduction to recommender systems handbook. Springer, 2011.

[2] G. Adomavicius and Y. Kwon, "New recommendation techniques for multicriteria rating systems," IEEE Intelligent Systems, vol. 22, no. 3, pp. 48-55, 2007.

[3] Y. Hu, Q. Peng, X. Hu, and R. Yang, "Time aware and data sparsity tolerant web service recommendation based on improved collaborative filtering," IEEE Transactions on Services Computing, vol. 8, no. 5, pp. 782-794, 2015.

[4] J. L. Herlocker, J. A. Konstan, and J. Riedl, "Explaining collaborative filtering recommendations," in Proceedings of the 2000 ACM Conference on Computer Supported Cooperative Work, ser. CSCW '00, 2000, pp. 241-250.

[5] L. C. Wang, X. Y. Zeng, L. Koehl, and Y. Chen, "Intelligent fashion recommender system: Fuzzy logic in personalized garment design," IEEE Transactions on Human-Machine Systems, vol. 45, no. 1, pp. 95-109, 2015.

[6] N. Y. Asabere, F. Xia, W. Wang, J. J. P. C. Rodrigues, F. Basso, and J. Ma, "Improving smart conference participation through socially aware recommendation," IEEE Transactions on Human-Machine Systems, vol. 44, no. 5, pp. 689-700, 2014.

[7] M. A. Domingues, M. G. Manzato, R. M. Marcacini, C. V. Sundermann, and S. O. Rezende, "Using contextual information from topic hierarchies to improve context-aware recommender systems," in 2014 22nd International Conference on Pattern Recognition (ICPR). IEEE, 2014, pp. 3606-3611.

[8] B. Lika, K. Kolomvatsos, and S. Hadjiefthymiades, "Facing the cold start problem in recommender systems," Expert Systems with Applications, vol. 41, no. 4, Part 2, pp. 2065 - 2073, 2014.

[9] G. Guo, J. Zhang, and N. Yorke-Smith, "Leveraging multiviews of trust and similarity to enhance clustering-based recommender systems," Knowledge-based Systems, vol. 74, pp. 14-27, 2015.

[10] H. Oufadia and O. Nouali, "Exploiting semantic web technologies for recommender systems: A multiview recommendation engine," Proceedings of the 7th Workshop on Intelligent Techniques for Web Personalization Recommender Systems (ITWP), 2009.

[11] G. Adomavicius and A. Tuzhilin, Context-Aware Recommender Systems. In F. Ricci (Ed.): Recommender Systems Handbook. Springer US, 2011, pp. 217-253.

[12] I. Palomares, F. Browne, H. Wang, and P. Davis, "A collaborative filtering recommender system model using owa and uninorm aggregation operators," in Intelligent Systems and Knowledge Engineering (ISKE), 2015 10th International Conference on, 2015 , pp. 382-388. 
[13] L. H. Son, "Dealing with the new user cold-start problem in recommender systems: A comparative review," Information Systems, vol. 58, pp. $87-104,2016$

[14] I. Niskanen, M. Murtonen, F. Browne, P. Davis, and F. Pantisano, "A semantic layer for urban resilience content management," in $e K N O W$ 2016 : The Eighth International Conference on Information, Process, and Knowledge Management, IARIA, Ed., vol. 1, 2016, pp. 85-90.

[15] L. Zadeh, "Fuzzy sets," Information and Control, vol. 8, no. 3, pp. 338-353, 1965.

[16] R. Yera and L. Martinez, "Fuzzy tools in recommender systems: a survey," vol. 10, pp. 776-803, 2017.

[17] W. Wang, J. Lu, and G. Zhang, A New Similarity Measure-Based Collaborative Filtering Approach for Recommender Systems. In Foundations of Intelligent Systems: Proceedings of the Eighth International Conference on Intelligent Systems and Knowledge Engineering, Shenzhen, China, Nov 2013 (ISKE 2013), 2014, pp. 443-452.

[18] S. Meerow, J. P. Newell, and M. Stults, "Defining urban resilience: A review," Landscape and Urban Planning, vol. 147, pp. 38-49, 2016.

[19] I. Niskanen, M. Murtonen, F. Browne, P. Davis, and I. Palomares, "An integrated semantic approach to content management in the urban resilience domain," International Journal of Advances in Software, vol. 9, pp. 323-333, 2016.

[20] P. Lops, M. De Gemmis, and G. Semeraro, "Content-based recommender systems: State of the art and trends," in Recommender systems handbook. Springer, 2011, pp. 73-105.

[21] J. Castro, R. Rodríguez, and M. Barranco, "Weighting of features in content-based filtering with entropy and dependence measures," International Journal of Computational Intelligence Systems, vol. 7, no. 1, pp. 80-89, 2014.

[22] M. Ekstrand, J. Riedl, and J. Konstan, "Collaborative filtering recommender systems," Human-Computer Interaction, vol. 4, no. 2, pp. 81-173, 2010.

[23] J. B. Schafer, D. Frankowski, J. Herlocker, and S. Sen, "Collaborative filtering recommender systems," in The adaptive web. Springer, 2007, pp. 291-324.

[24] R. Burke, Hybrid Web Recommender Systems. Berlin, Heidelberg: Springer Berlin Heidelberg, 2007, pp. $377-408$.

[25] M. G. Vozalis and K. G. Margaritis, "Using svd and demographic data for the enhancement of generalized collaborative filtering," Information Sciences, vol. 177, no. 15, pp. 3017-3037, 2007.

[26] J. Castro, F. J. Quesada, I. Palomares, and L. Martínez, “A consensus-driven group recommender system,” International Journal of Intelligent Systems, vol. 30, no. 8, pp. 887-906, 2015.

[27] B. Sarwar, G. Karypis, J. Konstan, and J. Riedl, "Item-based collaborative filtering recommendation algorithms," in Proceedings of the 10th international conference on World Wide Web. ACM, 2001, pp. 285-295.

[28] J. S. Breese, D. Heckerman, and C. Kadie, "Empirical analysis of predictive algorithms for collaborative filtering," in Proceedings of the Fourteenth conference on Uncertainty in artificial intelligence. Morgan Kaufmann Publishers Inc., 1998, pp. 43-52.

[29] C. Aggarwal, Recommender Systems: The Textbook. Springer, 2016.

[30] L. Safoury and A. Salah, "Exploiting user demographic attributes for solving cold-start problem in recommender system," Lectures Notes in Software Engineering, vol. 1, no. 3, pp. 303-307, 2013.

[31] M. Doumpos and E. Grigoroudis, Multicriteria Decision Aid and Artificial Intelligence. Wiley, 2013.

[32] G. Beliakov, T. Calvo, and S. James, "Aggregation of preferences in recommender systems," in Recommender Systems Handbook. Springer, 2011.

[33] G. Beliakov, A. Pradera, and T. Calvo, Aggregation Functions: A Guide for Practitioners. Springer, 2007.

[34] I. J. Rudas, E. Pap, and J. Fodor, "Information aggregation in intelligent systems: An application oriented approach," Knowledge-Based Systems, vol. 38, pp. 3 - 13, 2013, special Issue on Advances in Fuzzy Knowledge Systems: Theory and Application.

[35] R. Yager, "On ordered weighted averaging aggregation operators in multi-criteria decision making," IEEE Transactions on Systems, Man and Cybernetics, vol. 18, no. 1, pp. 183-190, 1988.

[36] M. Roubens, "Fuzzy sets and decision analysis," Fuzzy Sets and Systems, vol. 90, no. 2, pp. 199-206, 1997.

[37] R. Yager, "Families of OWA operators," Fuzzy Sets and Systems, vol. 59, pp. 125-148, 1993.

[38] _ - "Quantifier guided aggregation using OWA operators," International Journal of Intelligent Systems, vol. 11, pp. 49-73, 1996.

[39] J. Fodor, J.-L. Marichal, and M. Roubens, "Characterization of the ordered weighted averaging operators," IEEE Transactions on Fuzzy Systems, vol. 3, no. 2, pp. 236-240, 1995.

[40] R. Yager and A. Rybalov, "Uninorm aggregation operators," Fuzzy Sets and Systems, vol. 80, pp. 111-120, 1996.

[41] J. Fodor, R. Yager, and A. Rybalov, "Structure of uninorms," International Journal of Uncertainty, Fuzziness and Knowledge-based Systems, vol. 5, pp. 411-427, 1997.

[42] X. Luo, Q. Zhong, and H.-f. Leung, "A spectrum of weighted compromise aggregation operators: A generalization of weighted uninorm operator," International Journal of Intelligent Systems, vol. 30, no. 12, pp. 1185-1226, 2015.

[43] G. Adomavicius and A. Tuzhilin, "Toward the next generation of recommender systems: a survey of the state-of-the-art and possible extensions," IEEE Transactions on Knowledge and Data Engineering, vol. 17, no. 6, pp. 734-749, 2005.

[44] L. Zadeh, "A computational approach to fuzzy quantifiers in natural languages," Computing and Mathematics with Applications, vol. 9, no. 1, pp. 149-184, 1983. 